\title{
The Obesity-Related Gut Bacterial and Viral Dysbiosis Can Impact the Risk of Colon Cancer Development
}

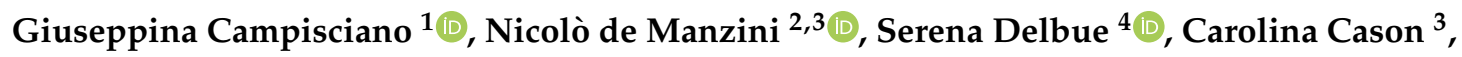 \\ Davide Cosola $^{2}$, Giuseppe Basile ${ }^{5}$, Pasquale Ferrante ${ }^{4}$, Manola Comar ${ }^{1,3, *}$ and \\ Silvia Palmisano ${ }^{2,3}$ \\ 1 SSD of Advanced Microbiology Diagnosis and Translational Research, Institute for Maternal and Child \\ Health-IRCCS “Burlo Garofolo”, Via dell'Istria 65/1, 34137 Trieste, Italy; giusi.campisciano@burlo.trieste.it \\ 2 General Surgery Clinic, Azienda Sanitaria Universitaria Giuliano Isontina (ASU GI), Strada di Fiume 447, \\ 34149 Trieste, Italy; ndemanzini@units.it (N.d.M.); d.cosola84@gmail.com (D.C.); spalmisano@units.it (S.P.) \\ 3 Department of Medical, Surgical and Health Sciences, Azienda Sanitaria Universitaria Giuliano \\ Isontina (ASU GI), Strada di Fiume 447, 34149 Trieste, Italy; casoncarolina@gmail.com \\ 4 Department of Biomedical, Surgical and Dental Sciences, Laboratory of Translational Research, Via Carlo \\ Pascal 36, 20133 Milano, Italy; serena.delbue@unimi.it (S.D.); pasquale.ferrante@unimi.it (P.F.) \\ 5 Clinical Institute San Siro, Via Monreale 18, 20148 Milano, Italy; giuseppe.basile@unife.it \\ * Correspondence: manola.comar@burlo.trieste.it; Tel.: +39-040-3785527
}

Received: 9 February 2020; Accepted: 17 March 2020; Published: 19 March 2020

\begin{abstract}
An incorrect food regimen from childhood is suggested to negatively impact the gut microbiome composition leading to obesity and perhaps to colon rectal cancer (CRC) in adults. In this study, we show that the obesity and cancer gut microbiota share a characteristic microbial profile with a high colonization by mucin degraders species, such as Hafnia alvei and Akkermansia muciniphila. In addition, the species Clostridium bolteae, a bacterium associated with insulin resistance, dyslipidemia, and inflammation, has been associated with the presence of oncogenic Human Polyomaviruses (HPyVs). Merkel cell Polyomavirus (MCPyV) and BK Polyomavirus (BKPyV) were the most frequently oncogenic viruses recovered in the gut of both obese and tumor patients. Considering the high seroprevalence of HPyVs in childhood, their association with specific bacterial species deserve to be further investigated. Data from the present study highlight the presence of a similar microbiome pattern in CRC and obese subjects, suggesting that obese microbiome may represent an opportunity for tumorigenic/driver bacteria and viruses to trigger cell transformation.
\end{abstract}

Keywords: microbiome; cancer pathogenesis; dysbiotic microbiota; obesity; gut microbiota

\section{Introduction}

Colorectal cancer (CRC) is a major public health concern, as it is one of the leading causes of cancer deaths in the Western world [1]. CRC requires decades to clinically manifest, starting from the initial transformed intestinal crypt cells [2]. Thus, this means that some risk factors could be traced back to the childhood and early adulthood. Especially the increase in the CRC incidence is considered a consequence of the modern life-style and is associated with calorically excessive high-fat/low-fiber diet, consumption of refined products, lack of physical activity, and obesity [3,4]. This association seems to be stronger when childhood overweight persists into early adulthood than when the overweight disappears before early adulthood or develop after childhood [5].

An unhealthy diet impacts gut microbial composition, triggering oncogenic transformation, conducive to the alteration of metabolic pathways, including insulin resistance, hyperinsulinemia, 
unbalanced level of growth factors, adipocytokines and steroid hormones [6]. Therefore, the time of exposure to these metabolic changes, especially if present since childhood, may increase the chances for the development of CRC in adulthood.

As of yet, a strong universal obesity-related microbial profile linked to CRC development has not been identified, due to the interpersonal variability driving CRC, including genetic factors, behavioral style, and diet. Nonetheless, the CRC 'driver-passenger' model suggests that symbiotic 'driver' bacteria initiate tissue malignant transformation through cell DNA damage and that colorectal tumorigenesis is successively mediated by alterations of the intestinal microenvironment, conducive to the proliferation of "passengers" opportunistic pathogens, such as Fusobacterium spp., Streptococcus bovis and Roseburia spp. [7]. Among "passengers" players, Human Polyoma Viruses (HPyVs) can also be mentioned. Interestingly, despite their known transformative abilities, HPyVs infections are often asymptomatic, with an age profile indicating high frequency of early-age infections and lifelong persistence [8-10].

To note, gut bacterial and viral dysbiosis in both CRC and obese patients are strongly responsible for altered host immune response, leading to a chronic inflammatory status [11].

The importance of resident bacteria in regulating the gut homeostasis has been reported for a group of gut colonizer microorganisms. Specifically, the alteration of quantitative relationship between bacterial composition in the intestinal lumen and mucosa has been described in patients with CRC, characterized by a decreased rate of colonization of Bifidobacterium, Faecalibacterium, and Blautia and the predominance of Porphyromonas and Mogibacterium species [12]. Moreover, the presence of "not gut" bacteria including Fusobacterium nucleatum and Fusobacterium necrophorum, commonly inhabiting the oral cavity, and occasionally causing periodontal and gingival infections, have been found repeatedly associated with CRC $[13,14]$.

At the same time, metagenomics analysis of gut of obese subjects showed an inverse ratio of Bacteroidetes to Firmicutes family, with high level of Lactobacillus species and with a relatively low level of Bacteroides [15].

Considering the dramatic increase of worldwide childhood obesity [16], understanding whether bacterial dysbiosis due to prolonged unhealthy diet, leading to obesity, plays a role in driving a more susceptible gut environment for CRC would represent an urgency in order to draw targeted preventive diet recommendations, starting from the early stage of life. In this regard, we wanted to assess whether the gut bacterial composition of obese patients, who were on an unhealthy diet from childhood, resembles that of patients affected with CRC.

\section{Materials and Methods}

\subsection{Case Study}

Stool samples were collected before surgery, as requested by the clinical practice, from 53 patients with CRC, eligible for surgery (Tumor Group, age $74 \pm 11$ ), and from 25 obese subjects, eligible for bariatric surgery (Obese Group, age 44,5 $\pm 9,5$ ), present at the Azienda Sanitaria Universitaria Giuliano Isontina (ASU GI), Trieste, Italy. Additionally, 27 healthy normal weight volunteer subjects (Control Group, age 44,2 $\pm 9,3$ ) were enrolled in the study. Clinical procedures have been completed in accordance with Good Clinical Practices and the ethical standards according to the Declaration of Helsinki.

Inclusion criteria for obese and control patients were in line with international bariatric guidelines [17]. Inclusion criteria for patients with CRC were primarily based on patients' willingness to participate in the study. Informed consent was obtained from all participants and the privacy rights of human subjects were observed. Exclusion criteria were familiarity for colorectal cancer, the presence of inflammatory bowel diseases and administration of antibiotic/probiotic therapy within 1 month before the study enrollment. 


\section{2. $16 S$ rRNA Gene Library Preparation Targeted Sequencing}

The NucliSENS ${ }^{\circledR}$ easyMAG system (bioMèrieux, Marcy l'Etoile, France) was used for nucleic acid extraction. In brief, $100 \mathrm{mg}$ of fecal sample were incubated with $1.8 \mathrm{~mL}$ of Lysis Buffer easyMAG and $50 \mu \mathrm{g} / \mathrm{mL}$ of proteinase $\mathrm{K}$ (Euroclone, Trieste, Italy), vortexed until the fecal sample was thoroughly homogenized and incubated at $56^{\circ} \mathrm{C}$ for $3 \mathrm{~h}$ with continuous shaking. The samples were centrifuged for $15 \mathrm{~min}$ at $13,000 \times \mathrm{g}$ and $500 \mu \mathrm{L}$ of the supernatant were used for the extraction, with an elution volume of $50 \mu \mathrm{L}$.

An EVAGREEN (EvaGreen ${ }^{\circledR}$ dye, Fisher Molecular Biology, Waltham, MA, USA) real-time polymerase chain reaction (PCR) using the 27FYM degenerated primer (5'-AGR GTT YGA TYM TGG CTC AG-3') and the U534R primer (targeting the V1-V3 region of $500 \mathrm{bp}$ length) was performed. A nested PCR was performed with the primers B338F_P1-Ion-adaptor (B338F 5'-ACTCCTACG GGAGGCAGC-3') and U534R_A-Ion-adaptor_IonXpress-barcode (U534R 5' -ATTACCGCGGCTG CTGG-3'), in order to amplify the V3-region template of 200 bases for sequencing analysis. Negative controls including no template were processed with clinical samples. The Kapa 2G HiFi Hotstart ready mix 2X (Kapa Biosystems, Wilmington, Massachusetts, USA) was used for the amplifications and $400 \mathrm{ng} / \mu \mathrm{L}$ BSA were added to the reaction mix. The temperature cycling was $5 \mathrm{~min}$ at $95^{\circ} \mathrm{C}, 30 \mathrm{~s}$ at $95^{\circ} \mathrm{C}, 30 \mathrm{~s}$ at $59^{\circ} \mathrm{C}$ (for $\mathrm{V} 1-\mathrm{V} 3$ region) $/ 57^{\circ} \mathrm{C}$ (for $\mathrm{V} 3$ region), $45 \mathrm{~s}$ at $72{ }^{\circ} \mathrm{C}$ and a final elongation step at $72^{\circ} \mathrm{C}$ for $10 \mathrm{~min}$.

Qubit ${ }^{\circledR}$ 2.0 Fluorometer (Invitrogen, Carlsbad, CA, USA) was used for the quantification of the amount of dsDNA. An equal amount of each PCR reaction was pooled into a single batch and the pooled-library diluted to a concentration of $100 \mathrm{pM}$.

Template preparation was performed using the Ion PGM Hi-Q View kit on Ion OneTouch ${ }^{\mathrm{TM}} 2$ System (Life Technologies, Gran Island, NY, USA) and sequenced using the Ion PGM Hi-Q View sequencing kit (Life Technologies, Gran Island, NY, USA) by the Ion PGM ${ }^{\mathrm{TM}}$ System technology.

\subsection{Bioinformatics Analysis}

QIIME 1.9.1 software (Quantitative Insights into Microbial Ecology) was used to process the sequence data [18]. High quality $(Q>20)$ sequences with a minimum length of $150 \mathrm{bp}$ were retained for the analyses. Operational taxonomic units (OTUs) were assigned at $97 \%$ similarity and clustered against the Human Intestinal Database [19] using open-reference OTU picking [20] with a uclust clustering tool [21]. Before further analysis, singleton OTUs and samples with low sequencing depth were removed (less than 10,000 reads) and a cumulative sum scaling was applied.

\subsection{Detection of the HPyV Genomes}

Q-PCR reactions specific for the six HPyV analyzed (JCPyV, BKPyV, Merkel cell PyV-MCPyV-, HPyV-6, HPyV-7, and HPyV-9) were performed, using 1X Taqman Universal PCR Master Mix (Applied Biosystems, Foster City, CA, USA), 0,4 $\mu \mathrm{M}$ primer Forward, 0,4 to $0,9 \mu \mathrm{M}$ primer Reverse, $0,2 \mu \mathrm{M}$ probe, and $250 \mathrm{ng}$ of extracted nucleic acid, in a final volume of $25 \mu \mathrm{L}$ [22]. Thermal cycling was performed as follows: denaturation step at $95^{\circ} \mathrm{C}$ for $10 \mathrm{~min}$, followed by 40 cycles of $95^{\circ} \mathrm{C}$ for $15 \mathrm{~s}$ and $60^{\circ} \mathrm{C}$ for $1 \mathrm{~min}$, at the end of which the fluorescence was acquired. A 10-fold dilution series of plasmids containing the entire VP1 gene of BKPyV, MCPyV, HPyV-6, HPyV-7, and HPyV-9 and the entire LT of JCPyV (dilution range: 108-10 copies/ $\mu \mathrm{L}$ ) was used to construct the standard curves. The limit of detection of each assay was 10 copies/ $\mu \mathrm{L}$. To determine the quality and percentage of infected cells, a concomitant Q-PCR assay targeting the $\beta$-globin gene was performed on the same samples using the primer set and thermal cycles previously published [23].

Viral loads were expressed as copies/ $\mu$ g of DNA extracted. Negative and positive controls were included in each run. Each sample, standard and control, was tested in triplicate. The results were analyzed by the absolute quantification method and reported as copies $/ \mathrm{mL}$ and percentage of infected cells, calculated as follows: ([viral copies]/[beta-globin copies/2]) $\times 100$. 


\subsection{Statistical Analysis}

Differences in microbial community composition were investigated using QIIME 1.9.1. Chao1, Observed species, and Shannon metrics were used to assess alpha diversity (within-sample diversity), while the LEfSe test (Linear discriminant analysis Effect Size) for beta diversity (between sample diversity comparison). To test whether any individual bacterium specifically correlated with a clinical metadata (sex, age, body mass index (BMI), diabetes, smoke, alcohol, HPyVs), the observation_metadata_correlation.py script, using bootstrapping and Pearson correlation, was used. To test microbial differences between positive and negative samples for HPyVs, a non-parametric $\mathrm{T}$ Test was used. All the statistical analyses were performed after rarefying the otu_table.biom (depth 10,000 reads/sample) and applying a cumulative sum scaling.

\section{Results}

We performed the sequencing of the V3 region of the16S rRNA gene from 107 biological samples including 53 stool samples from CRC patients (Tumor group), 27 stool samples from obese patients (Obese group), and 27 healthy normal weight patients (Control group). Table 1 shows the patients' demographics. The two negative controls analyzed did not produce any sequencing output after the quality filtering. The sequencing of the remaining samples produced a total of 5206,621 reads (Q score $>20$, range 5519-160,737) and a total number of observed OTUs of 11,815 (the reads were clustered into $200 \pm 50$ OTUs per sample).

Table 1. Patients' demographics. Abbreviations: $\mathrm{m}=$ mean; $\mathrm{sd}=$ standard deviation; yrs = years; $\mathrm{T}=$ tumor; $\mathrm{O}=$ Obese; $\mathrm{C}=$ Control; $\mathrm{T} 2 \mathrm{DM}=$ type 2 diabetes mellitus.

\begin{tabular}{|c|c|c|c|c|}
\hline & Tumor & Obese & Control & Fisher's Exact Test $p$ Value \\
\hline Age $(m \pm s d)$ & $74 \pm 11 \mathrm{yrs}$ & $44 \pm 9$ yrs & $44 \pm 9$ yrs & $p<0.001:$ T vs. $\mathrm{O} / \mathrm{C}$ \\
\hline Women & 21 & 21 & 20 & \multirow{2}{*}{$p<0.001: \mathrm{T}$ vs. $\mathrm{O} / \mathrm{C}$} \\
\hline Men & 32 & 4 & 7 & \\
\hline $\mathrm{BMI}(\mathrm{m} \pm \mathrm{sd})$ & $26 \pm 6 \mathrm{~kg} / \mathrm{m}^{2}$ & $37 \pm 7 \mathrm{~kg} / \mathrm{m}^{2}$ & $22 \pm 3 \mathrm{~kg} / \mathrm{m}^{2}$ & $\begin{array}{c}p<0.001: \mathrm{O} \text { vs. T/C; } \\
p<0.05: \mathrm{T} \text { vs. } \mathrm{C}\end{array}$ \\
\hline Smoke & $13 / 53(24 \%)$ & $9 / 25(36 \%)$ & $3 / 27(11 \%)$ & - \\
\hline Alcohol & $8 / 53(15 \%)$ & 0 & 0 & $p<0.05:$ T vs. $\mathrm{O} / \mathrm{C}$ \\
\hline T2DM & $17 / 53(32 \%)$ & $1 / 25(4 \%)$ & 0 & $p<0.05: \mathrm{C}$ vs. $\mathrm{O} / \mathrm{T}$ \\
\hline
\end{tabular}

\subsection{Alpha Diversity}

Alpha diversity was quantified by Chao1 diversity index, by the total number of observed species, and by Shannon index. Table 2 shows the alpha diversity measurements for Tumor, Obese, and Control groups. Statistical testing showed no difference for Chao1 richness estimator and the observed species ( $p$ value $>0.05$ ). Nevertheless, a decrease trend of alpha diversity was observed in the Tumor group for Chao1 and Observed species metrics.

Shannon, which relates both OTU richness and evenness, did not reveal a significant change. However, the Tumor group showed a slight decrease trend towards the value of the Obese group (Table 2). 
Table 2. Alpha diversity. Bacterial diversity values are given as mean \pm standard deviation at a rarefaction depth of 10,000 sequences per sample. Alpha diversity was compared between pairwise comparisons by means of a non-parametric t-test using the compare_alpha_diversity.py script of QIIME. None significant variation was observed. Abbreviations: $\mathrm{T}=$ tumor; $\mathrm{O}=$ obese $\mathrm{C}=\mathrm{Control}$.

\begin{tabular}{ccccccc}
\hline Metric & Tumor & Obese & Control & $\begin{array}{c}p \text { Value } \\
\text { T vs. O }\end{array}$ & $\begin{array}{c}p \text { Value } \\
\text { T vs. C }\end{array}$ & $\begin{array}{c}p \text { Value } \\
\text { O vs. C }\end{array}$ \\
\hline Chao1 & $559 \pm 187$ & $662 \pm 201$ & $642 \pm 248$ & 0.1 & 0.3 & 1 \\
\hline Observed species & $289 \pm 75$ & $313 \pm 72$ & $329 \pm 113$ & 0.6 & 0.2 & 1 \\
\hline Shannon & $4.9 \pm 0.6$ & $4.7 \pm 0.5$ & $5 \pm 0.8$ & 0.3 & 1 & 0.3 \\
\hline
\end{tabular}

\subsection{Specific OTUs}

The observation_metadata_correlation.py script did not reveal any significant association of specific bacteria with the clinical metadata, such as diabetes, alcohol, smoke, BMI, sex, and HPyV. Thus, excluding cofounding factors in the observed differences between clinical groups.

Next, the LEfSe test was used in order to identify significantly imbalanced biomarkers, which showed the strongest effects for group differentiation. Analysis at the phylum level showed that Verrucomicrobia ( $p$ value 0.004 , LDA score 4.8 ) were significantly higher in the Tumor group (Table 3 ).

Table 3. Specific phyla. Linear discriminative analysis (LDA) effect size (LEfSe) test showing the phyla associated with the three groups (Tumor, Obese, Control). Positive values of LDA scores (log 10) are indicative for enriched taxa in a given group. Significant $p$ values are highlighted in bold. Abbreviations: $\mathrm{sd}=$ standard deviation; $\mathrm{FDR}=$ false discovery rate.

\begin{tabular}{ccccccccc}
\hline Bacterial Phyla & \multicolumn{2}{c}{ Tumor } & \multicolumn{2}{c}{ Obese } & \multicolumn{2}{c}{ Control } & \multirow{2}{*}{$\begin{array}{c}\text { Value } \\
\text { FDR }\end{array}$} & \multirow{2}{*}{ LDA Score } \\
\hline & Mean & sd & Mean & sd & Mean & sd & 5.1 \\
Actinobacteria & $1.29 \%$ & $1.94 \%$ & $3.42 \%$ & $7.17 \%$ & $2.65 \%$ & $4.90 \%$ & 0.38 \\
Bacteroidetes & $53.08 \%$ & $16.11 \%$ & $59.33 \%$ & $21.79 \%$ & $56.41 \%$ & $18.82 \%$ & 0.33 & 5.5 \\
Firmicutes & $32.88 \%$ & $13.29 \%$ & $30.14 \%$ & $14.91 \%$ & $35.75 \%$ & $16.68 \%$ & 0.41 & 5.4 \\
Fusobacteria & $0.33 \%$ & $1.16 \%$ & $0.33 \%$ & $1.17 \%$ & $0.01 \%$ & $0.06 \%$ & 0.22 & 4.1 \\
Lentisphaerae & $0.06 \%$ & $0.16 \%$ & $0.01 \%$ & $0.03 \%$ & $0.03 \%$ & $0.07 \%$ & 0.38 \\
Proteobacteria & $10.83 \%$ & $11.96 \%$ & $6.08 \%$ & $7.70 \%$ & $4.74 \%$ & $5.57 \%$ & 0.11 \\
Synergistetes & $0.29 \%$ & $0.89 \%$ & $0.14 \%$ & $0.71 \%$ & $0.10 \%$ & $0.44 \%$ & 0.11 & 2.8 \\
Verrucomicrobia & $1.10 \%$ & $2.13 \%$ & $0.07 \%$ & $0.19 \%$ & $0.03 \%$ & $0.06 \%$ & $\mathbf{0 . 0 0 4}$ & 4.0 \\
\hline
\end{tabular}

At the species level, the increased amount of Proteobacteria and Verrucomicrobia in the Tumor group were dependent mainly on the increase of Hafnia alvei $(7 \% \pm 11 \%)$, which had the highest LDA score, and Akkermansia muciniphila $(1 \% \pm 2 \%)$, respectively. Among Bacteroidetes, there was a different distribution of species among groups, with Alistipes senegalensis higher in the Tumor group $(1.5 \% \pm 3 \%)$ while Barnesiella intestinihomins was higher in the Control group $(0.11 \% \pm 0.18 \%)$. Among Firmicutes, several species were identified as biomarkers by LEfSe test. Among these, Blautia wexlerae, Eubacterium rectale, Lactobacillus rogosae, and Ruminococcus faecis showed a similar relative abundance between Tumor and Obese groups (Table 4). 
Table 4. Specific species. Linear discriminative analysis (LDA) effect size (LEfSe) test showing the species associated with the three groups (Tumor, Obese, Control). Positive values of LDA scores (log 10) are indicative for enriched taxa in a given group. Significant $p$ values are highlighted in bold. Abbreviations: $\mathrm{m}=$ mean; $\mathrm{sd}=$ standard deviation; $\mathrm{FDR}=$ false discovery rate; $B=$ Bacteroidetes; $F=$ Firmicutes $P=$ Proteobacteria $; V=$ Verrucomicrobia .

\begin{tabular}{|c|c|c|c|c|c|c|c|c|c|}
\hline \multirow[b]{2}{*}{ Phylum } & \multirow[b]{2}{*}{ Species } & \multicolumn{2}{|c|}{ Tumor } & \multicolumn{2}{|c|}{ Obese } & \multicolumn{2}{|c|}{ Control } & \multirow{2}{*}{$\begin{array}{l}p \text { Value } \\
\text { FDR }\end{array}$} & \multirow[b]{2}{*}{$\begin{array}{l}\text { LDA } \\
\text { Score }\end{array}$} \\
\hline & & $\mathbf{M}$ & sd & m & sd & $\mathbf{m}$ & sd & & \\
\hline \multirow[t]{3}{*}{$B$} & Alistipes putredinis & $3.03 \%$ & $3.02 \%$ & $0.88 \%$ & $1.41 \%$ & $3.02 \%$ & $2.81 \%$ & 0.03 & 5.0 \\
\hline & $\begin{array}{c}\text { Alistipes } \\
\text { senegalensis }\end{array}$ & $1.54 \%$ & $3.11 \%$ & $0.41 \%$ & $0.92 \%$ & $0.52 \%$ & $0.87 \%$ & 0.02 & 4.8 \\
\hline & $\begin{array}{c}\text { Barnesiella } \\
\text { intestinihominis }\end{array}$ & $0.05 \%$ & $0.11 \%$ & $0.02 \%$ & $0.04 \%$ & $0.11 \%$ & $0.18 \%$ & 0.03 & 2.9 \\
\hline \multirow[t]{9}{*}{$F$} & Blautia wexlerae & $0.25 \%$ & $1.38 \%$ & $0.46 \%$ & $1.10 \%$ & $0.84 \%$ & $3.01 \%$ & 0.02 & 4.5 \\
\hline & $\begin{array}{l}\text { Christensenella } \\
\text { minuta }\end{array}$ & $0.20 \%$ & $0.64 \%$ & $0.00 \%$ & $0.00 \%$ & $0.11 \%$ & $0.27 \%$ & 0.03 & 4.0 \\
\hline & $\begin{array}{l}\text { Clostridium } \\
\text { bartlettii }\end{array}$ & $0.32 \%$ & $0.79 \%$ & $1.90 \%$ & $4.29 \%$ & $0.29 \%$ & $0.74 \%$ & 0.05 & 4.9 \\
\hline & Clostridium bolteae & $0.01 \%$ & $0.01 \%$ & $0.03 \%$ & $0.08 \%$ & $0.01 \%$ & $0.02 \%$ & 0.00 & 4.6 \\
\hline & $\begin{array}{l}\text { Clostridium } \\
\text { clariflavum }\end{array}$ & $0.10 \%$ & $0.22 \%$ & $0.00 \%$ & $0.01 \%$ & $0.22 \%$ & $0.40 \%$ & 0.03 & 4.1 \\
\hline & Eubacterium rectale & $0.03 \%$ & $0.05 \%$ & $0.10 \%$ & $0.15 \%$ & $0.35 \%$ & $0.57 \%$ & 0.02 & 4.2 \\
\hline & Lactobacillus rogosae & $0.10 \%$ & $0.15 \%$ & $0.11 \%$ & $0.19 \%$ & $0.43 \%$ & $0.54 \%$ & 0.02 & 4.3 \\
\hline & $\begin{array}{l}\text { Papillibacter } \\
\text { cinnamivorans }\end{array}$ & $0.16 \%$ & $0.32 \%$ & $0.01 \%$ & $0.02 \%$ & $0.38 \%$ & $0.96 \%$ & 0.03 & 4.3 \\
\hline & Ruminococcus faecis & $0.12 \%$ & $0.42 \%$ & $0.12 \%$ & $0.58 \%$ & $0.08 \%$ & $0.17 \%$ & 0.03 & 3.7 \\
\hline \multirow[t]{3}{*}{$P$} & Hafnia alvei & $7.81 \%$ & $11.15 \%$ & $1.83 \%$ & $6.82 \%$ & $0.78 \%$ & $1.49 \%$ & 0.00 & 5.6 \\
\hline & $\begin{array}{c}\text { Parasutterella } \\
\text { excrementihominis }\end{array}$ & $0.13 \%$ & $0.41 \%$ & $1.21 \%$ & $2.90 \%$ & $1.19 \%$ & $2.76 \%$ & 0.02 & 4.7 \\
\hline & $\begin{array}{l}\text { Yokenella } \\
\text { regensburgei }\end{array}$ & $0.13 \%$ & $0.50 \%$ & $0.02 \%$ & $0.07 \%$ & $0.00 \%$ & $0.00 \%$ & 0.03 & 3.8 \\
\hline$V$ & $\begin{array}{l}\text { Akkermansia } \\
\text { muciniphila }\end{array}$ & $1.10 \%$ & $2.13 \%$ & $0.07 \%$ & $0.19 \%$ & $0.03 \%$ & $0.06 \%$ & 0.01 & 4.8 \\
\hline
\end{tabular}

\subsection{Microbiome Composition of HPyV-Positive Samples}

Overall, HPyV genomes were detected in seven stool samples. Of the six searched HPyVs, only $\mathrm{BKPyV}$ and MCPyV were detected. Precisely, BKPyV was identified in 2/53 (3.8\%) of stool samples from the Tumor group, while MCPyV was equally distributed between samples from Tumor $(4 / 53 ; 7.6 \%)$ and Obese groups (2/27; 7.4\%). One out of $27(3.7 \%)$ samples from the Control group showed MCPyV infection (Table 5).

Comparing the microbiome profile of the HPyV positive with the HPyV negative samples by a non-parametric $\mathrm{T}$ test, using the rarefied biom table (10,000 reads/sample), some differences were observed. Precisely, a significant $(p$ value $=0.014$ ) higher relative abundance of Clostridium bolteae was observed in HPyVs positive samples comparing with negative samples $(0.27 \% \pm 0.63 \%$ vs. $0.07 \% \pm 0.17$, respectively).

Table 5. HPyVs prevalence among patients.

\begin{tabular}{ccccccc}
\hline Virus & Tumor (53) & $\begin{array}{c}\text { Mean Viral } \\
\text { Load } \\
\text { (Copies/mL) }\end{array}$ & Control (27) & $\begin{array}{c}\text { Mean Viral } \\
\text { Load } \\
\text { (Copies/mL) }\end{array}$ & Obese (27) & $\begin{array}{c}\text { Mean Viral } \\
\text { Load } \\
\text { (Copies/mL) }\end{array}$ \\
\hline $\begin{array}{c}\text { BKPyV+/tot } \\
(\%)\end{array}$ & $\begin{array}{c}2 / 53 \\
(3.8 \%)\end{array}$ & $6 \times 10^{3}$ & $0 / 27$ & & $0 / 27$ & \\
\hline $\begin{array}{c}\text { MCPyV+/tot } \\
(\%)\end{array}$ & $4 / 53$ & $4.2 \times 10^{5}$ & $\begin{array}{c}1 / 27 \\
(3.7 \%)\end{array}$ & $8 \times 10^{4}$ & $2 / 27$ & $3.3 \times 10^{4}$ \\
\hline $\begin{array}{c}\text { HPyV+/tot } \\
(\%)\end{array}$ & $6 / 53$ & $2.1 \times 10^{5}$ & $\begin{array}{c}1 / 27 \\
(3.7 \%)\end{array}$ & $8 \times 10^{4}$ & $2 / 27$ & $3.3 \times 10^{4}$ \\
\hline
\end{tabular}




\section{Discussion}

Data from the present study suggest a similar composition of the gut microbiome from CRC and obese patients. It is plausible to assert that dysbiosis usually observed in obese people [24] represents an opportunity for "tumorigenic/driver bacteria" to proliferate, and to elicit a low-grade persistent mucosal inflammation which turns into cell damage. Thus, as long as the obesity condition persists, it is more likely for an oncogenic transformation to take place, especially when the unhealthy diet starts from childhood.

We compared the gut microbiota of the CRC group with the gut dysbiotic microbiota of obese subjects, with a known history of unhealthy diet and obesity since childhood, and with the gut eubiotic microbiota of normal-weight subjects. Our aim was to highlight overlapping microbial signatures that can lead to an increased understanding of the bacterial activity in the pathogenesis of CRC. These shared microbial signatures can be of great impact to define a tailored preventive dysbiosis correction since the early stage of life and especially in childhood obesity causing long-term consequences on gut microbial composition.

The alpha diversity of the gut microbiome from the three groups did not significantly differ. This is plausible considering that in the gut there is always an equilibrium in which one phylum increases at the expense of another one, without affecting the total number of microorganisms. Indeed, the Tumor group showed only a slight decrease of bacterial diversity, but, at the same time, showing a peculiar bacterial composition, suggestive of a role for microorganism in CRC. The CRC gut microbiota showed higher abundance of Proteobacteria and Verrucomicrobia, phyla usually present at low amount with respect to Firmicutes and Bacteroidetes in the gut $[25,26]$. The same increase trend was observed in the Obese group, suggesting a similar dysbiotic gut picture.

Most notably, this overlapping was confirmed at the species level. Within the two Proteobacteria and Verrucomicrobia phyla, we observed the increase of Hafnia alvei (Proteobacteria) and Akkermansia muciniphila (Verrucomicrobia) in the Tumor and Obese groups. These bacteria are both mucin degraders and their increase is likely a consequence of the overexpression of the two types of mucins MUC1 and MUC5AC observed in patients affected with CRC [27-30]. These data suggest a strict interplay between genic expression of the host and the bacterial response, highlighting how an altered host milieu can affect the microbial signature that, in turn, leads to inflammation and tissue damage.

When considering the presence of possible risks factors, such as HPyVs infections, the presence of these viruses correlated with a higher amount of Clostridium bolteae $(0.27 \% \pm 0.63 \%$ in HPy $\mathrm{H}$ positive samples vs. $0.07 \% \pm 0.17 \%$ in negative samples). C. bolteae has been associated with insulin resistance, dyslipidemia and inflammation [31], which are typical aspects of obese people. Thus, in this case, the driver-passenger model can comprise the interaction between a bacterial species and HPyVs, with the inflammation-associated bacterium being present decades before CRC onset, in a dysbiotic gut environment, and the $\mathrm{HPyV}$ infection being an adjunctive risk factor. Considering the common and often asymptomatic prevalence of $\mathrm{HPyVs}$ in childhood, this interaction needs to be further investigated.

\section{Conclusions}

In conclusion, identifying similarities between obesity-associated gut microbiota and CRC-associated gut microbiota holds great promise to identify key microbiota members to target in order to restore a healthy environment and to suggest preventive diet recommendation from the early stage of life in order to reduce the risk of CRC development in adulthood.

Author Contributions: Conceptualization, G.C. and M.C.; methodology, G.C., C.C., and S.D.; resources, D.C. and S.P.; writing —original draft preparation, G.C. and M.C.; writing—review and editing, N.d.M., P.F., and G.B.; visualization, G.C.; supervision, M.C. All authors have read and agreed to the published version of the manuscript.

Funding: This research received no specific grant from any funding agency, commercial, or not-for-profit sectors.

Acknowledgments: We would like to thank the NGS Facility at the institute for maternal and child health "IRCCS Burlo Garofolo", Trieste, Italy. 
Conflicts of Interest: The authors declare no conflict of interest.

\section{References}

1. Bhandari, A.; Woodhouse, M.; Gupta, S. Colorectal cancer is a leading cause of cancer incidence and mortality among adults younger than 50 years in the USA: A seer-based analysis with comparison to other young-onset cancers. J. Investig. Med. 2017, 65, 311-315. [CrossRef] [PubMed]

2. Meza, R.; Jeon, J.; Renehan, A.G.; Luebeck, E.G. Colorectal cancer incidence trends in the united states and united kingdom: Evidence of right- to left-sided biological gradients with implications for screening. Cancer Res. 2010, 70, 5419-5429. [CrossRef] [PubMed]

3. Mehta, R.S.; Nishihara, R.; Cao, Y.; Song, M.; Mima, K.; Qian, Z.R.; Nowak, J.A.; Kosumi, K.; Hamada, T.; Masugi, Y.; et al. Association of dietary patterns with risk of colorectal cancer subtypes classified by fusobacterium nucleatum in tumor tissue. JAMA Oncol. 2017, 3, 921-927. [CrossRef] [PubMed]

4. Park, Y.; Hunter, D.J.; Spiegelman, D.; Bergkvist, L.; Berrino, F.; Van den Brandt, P.A.; Buring, J.E.; Colditz, G.A.; Freudenheim, J.L.; Fuchs, C.S.; et al. Dietary fiber intake and risk of colorectal cancer: A pooled analysis of prospective cohort studies. JAMA 2005, 294, 2849-2857. [CrossRef]

5. Jensen, B.W.; Bjerregaard, L.G.; Angquist, L.; Gogenur, I.; Renehan, A.G.; Osler, M.; Sorensen, T.I.A.; Baker, J.L. Change in weight status from childhood to early adulthood and late adulthood risk of colon cancer in men: A population-based cohort study. Int. J. Obes. 2018, 42, 1797-1803. [CrossRef]

6. Vernieri, C.; Casola, S.; Foiani, M.; Pietrantonio, F.; De Braud, F.; Longo, V. Targeting cancer metabolism: Dietary and pharmacologic interventions. Cancer Discov. 2016, 6, 1315-1333. [CrossRef]

7. Tjalsma, H.; Boleij, A.; Marchesi, J.R.; Dutilh, B.E. A bacterial driver-passenger model for colorectal cancer: Beyond the usual suspects. Nature reviews. Microbiology 2012, 10, 575-582. [CrossRef]

8. Kean, J.M.; Rao, S.; Wang, M.; Garcea, R.L. Seroepidemiology of human polyomaviruses. PLoS Pathog. 2009, 5, e1000363. [CrossRef]

9. Viscidi, R.P.; Rollison, D.E.; Sondak, V.K.; Silver, B.; Messina, J.L.; Giuliano, A.R.; Fulp, W.; Ajidahun, A.; Rivanera, D. Age-specific seroprevalence of merkel cell polyomavirus, bk virus, and jc virus. Clin. Vaccine Immunol. 2011, 18, 1737-1743. [CrossRef]

10. Baez, C.F.; Brandao Varella, R.; Villani, S.; Delbue, S. Human polyomaviruses: The battle of large and small tumor antigens. Virology 2017, 8, 1178122-17744785. [CrossRef]

11. Saltzman, E.T.; Palacios, T.; Thomsen, M.; Vitetta, L. Intestinal microbiome shifts, dysbiosis, inflammation, and non-alcoholic fatty liver disease. Front. Microbiol. 2018, 9, 61. [CrossRef] [PubMed]

12. Kelly, D.; Yang, L.; Pei, Z. Gut microbiota, fusobacteria, and colorectal cancer. Diseases 2018, 6, 109. [CrossRef] [PubMed]

13. Shang, F.M.; Liu, H.L. Fusobacterium nucleatum and colorectal cancer: A review. World J. Gastrointest. Oncol. 2018, 10, 71-81. [CrossRef] [PubMed]

14. Zhou, Z.; Chen, J.; Yao, H.; Hu, H. Fusobacterium and colorectal cancer. Front. Oncol. 2018, 8, 371. [CrossRef] [PubMed]

15. Ley, R.E.; Turnbaugh, P.J.; Klein, S.; Gordon, J.I. Microbial ecology: Human gut microbes associated with obesity. Nature 2006, 444, 1022-1023. [CrossRef] [PubMed]

16. Nishtar, S.; Gluckman, P.; Armstrong, T. Ending childhood obesity: A time for action. Lancet 2016, 387, 825-827. [CrossRef]

17. NIH conference. Gastrointestinal surgery for severe obesity. Consensus Development Conference Panel. Ann. Intern. Med. 1991, 115, 956-961.

18. Campisciano, G.; Palmisano, S.; Cason, C.; Giuricin, M.; Silvestri, M.; Guerra, M.; Macor, D.; De Manzini, N.; Croce, L.S.; Comar, M. Gut microbiota characterisation in obese patients before and after bariatric surgery. Benef. Microbes 2018, 9, 367-373. [CrossRef]

19. Thursby, E.; Juge, N. Introduction to the human gut microbiota. Biochem. J. 2017, 474, 1823-1836. [CrossRef]

20. Rinninella, E.; Raoul, P.; Cintoni, M.; Franceschi, F.; Miggiano, G.A.D.; Gasbarrini, A.; Mele, M.C. What is the healthy gut microbiota composition? A changing ecosystem across age, environment, diet, and diseases. Microorganisms 2019, 7, 14. [CrossRef] 
21. Janda, J.M.; Abbott, S.L.; Bystrom, S.; Probert, W.S. Identification of two distinct hybridization groups in the genus hafnia by 16s rrna gene sequencing and phenotypic methods. J. Clin. Microbiol. 2005, 43, 3320-3323. [CrossRef]

22. Derrien, M.; Collado, M.C.; Ben-Amor, K.; Salminen, S.; De Vos, W.M. The mucin degrader akkermansia muciniphila is an abundant resident of the human intestinal tract. Appl. Environ. Microbiol. 2008, 74, 1646-1648. [CrossRef] [PubMed]

23. Zeng, Y.; Zhang, Q.; Zhang, Y.; Lu, M.; Liu, Y.; Zheng, T.; Feng, S.; Hao, M.; Shi, H. Muc1 predicts colorectal cancer metastasis: A systematic review and meta-analysis of case controlled studies. PLoS ONE 2015, 10, e0138049. [CrossRef] [PubMed]

24. Wang, H.; Jin, S.; Lu, H.; Mi, S.; Shao, W.; Zuo, X.; Yin, H.; Zeng, S.; Shimamoto, F.; Qi, G. Expression of survivin, muc2 and muc5 in colorectal cancer and their association with clinicopathological characteristics. Oncol. Letters 2017, 14, 1011-1016. [CrossRef] [PubMed]

25. Lopetuso, L.R.; Petito, V.; Graziani, C.; Schiavoni, E.; Paroni Sterbini, F.; Poscia, A.; Gaetani, E.; Franceschi, F.; Cammarota, G.; Sanguinetti, M.; et al. Gut microbiota in health, diverticular disease, irritable bowel syndrome, and inflammatory bowel diseases: Time for microbial marker of gastrointestinal disorders. Dig. Dis. 2018, 36, 56-65. [CrossRef]

26. Caporaso, J.G.; Kuczynski, J.; Stombaugh, J.; Bittinger, K.; Bushman, F.D.; Costello, E.K.; Fierer, N.; Pena, A.G.; Goodrich, J.K.; Gordon, J.I.; et al. Qiime allows analysis of high-throughput community sequencing data. Nat. Methods 2010, 7, 335-336. [CrossRef]

27. Ritari, J.; Salojarvi, J.; Lahti, L.; De Vos, W.M. Improved taxonomic assignment of human intestinal 16s rrna sequences by a dedicated reference database. BMC Genom. 2015, 16, 1056. [CrossRef]

28. Rideout, J.R.; He, Y.; Navas-Molina, J.A.; Walters, W.A.; Ursell, L.K.; Gibbons, S.M.; Chase, J.; McDonald, D.; Gonzalez, A.; Robbins-Pianka, A.; et al. Subsampled open-reference clustering creates consistent, comprehensive otu definitions and scales to billions of sequences. PeerJ 2014, 2, e545. [CrossRef]

29. Edgar, R.C. Search and clustering orders of magnitude faster than blast. Bioinformatics 2010, 26, $2460-2461$. [CrossRef]

30. Zanotta, N.; Delbue, S.; Signorini, L.; Villani, S.; D’Alessandro, S.; Campisciano, G.; Colli, C.; De Seta, F.; Ferrante, P.; Comar, M. Merkel cell polyomavirus is associated with anal infections in men who have sex with men. Microorganisms 2019, 7, 54. [CrossRef]

31. Lo, Y.M.; Tein, M.S.; Lau, T.K.; Haines, C.J.; Leung, T.N.; Poon, P.M.; Wainscoat, J.S.; Johnson, P.J.; Chang, A.M.; Hjelm, N.M. Quantitative analysis of fetal DNA in maternal plasma and serum: Implications for noninvasive prenatal diagnosis. Am. J. Hum. Genet. 1998, 62, 768-775. [CrossRef] [PubMed] 